Volume 2, Issue 2, April-June 2017, Pages: 187, DOI: http://dx.doi.org/10.19082/ah187

\title{
DESIGN AND IMPLEMENTATION OF TABRIZ STROKE REGISTRY
}

\author{
Mehdi Farhoudi1, Reza Deljavan ${ }^{1}$, Homayoun Sadeghi-Bazargani², Faramarz Pour Asghar ${ }^{3}$, Masumeh Zamanlu ${ }^{1}$ \\ 1: Neuroscience Research Center, Tabriz University of Medical Sciences, Tabriz, Iran \\ 2: Neuroscience Research Center, Statistics and Epidemiology Department, Tabriz University of Medical Sciences, \\ Tabriz, Iran \\ 3: Faculty of Health, Tabriz University of Medical Sciences, Tabriz, Iran
}

Correspondence:

Reza Deljavan, Neuroscience Research Center, Tabriz University of Medical Sciences, Tabriz, Iran, Tel: +984133340730, Fax:+984133340730, E-mail: deljavan1111@yahoo.com

\section{TYPE OF ARTICLE: CONFERENCE ABSTRACT}

\begin{abstract}
Introduction: Stroke is a leading cause of mortality and disability worldwide; specifically, overwhelming in developing countries. Stroke registry is a beneficial infrastructure for clinical audits of stroke, as well as related surveillances, epidemiologic studies, evidence-based medicine and decisionmaking (both clinical and for health policies). However, few stroke registries have been designed and developed worldwide.

Methods: The structure of the stroke registry was designed by a thorough literature review of the stroke registries worldwide, assessed by an expert panel customized to Iranian culture and regional conditions in Tabriz. The software used for this registry is currently offline and expected to be online after specific progress of the registry. It is confirmed by the national council of stroke registries, and future stroke registries could join it in a national network. Security of information and ethical issues were considered. Results: Since 2014, Tabriz stroke registry is being implemented prospectively. Since now information of about 4000 stroke cases have been collected. The collected data include information of demography, pre-hospital, EMS (emergency medical service), clinical findings, primary imaging, lab findings, history of risk factors, drug history, discharge, follow up, GCS, MRS, NIHSS, dysphagia, rehabilitation, complications, instructions, diagnoses, and treatments (medical, surgical, interventional) of stroke patients. The collected data are being entered to the software and the registry is currently going on. Conclusion: Tabriz stroke registry has provided the efficient context for clinical and research promotion of stroke care which would influence this care in northwest Iran and eventually, the whole country.
\end{abstract}

KEYWORDS: Stroke registry, Registry implementation, Stroke epidemiology, Stroke surveillance

\footnotetext{
Abstracts of First National Congress of Medical Informatics, Mashhad, Iran, February 2017

(C) 2017 The Authors. This is an open access article under the terms of the Creative Commons Attribution-NonCommercialNoDerivs License, which permits use and distribution in any medium, provided the original work is properly cited, the use is non-commercial and no modifications or adaptations are made.
} 